\title{
Concomitent Chemotherapy and radiotherapy in patients withLocally Advanced Rectal Carcinoma
}

\author{
Mohamed Soliman Gaber Marzouk ${ }^{1}$, Mohamed Abo Elmagd Alhashemee ${ }^{2}$, \\ Elsayed Mostafa Ali ${ }^{3}$, Sahar Noor Eldeen Ahmed Hammam ${ }^{4}$ \\ (1)Prof. of Clinicl oncology ,faculty of medicine,Sohag University,(2) Prof. of \\ surgical oncology, south Egypt cancer Institute,(3) Assisstant Prof. of clinical \\ oncology, Faculty of medicine ,Sohag University,(4) Assisstant Lecturer of \\ clinical oncology Faculty of medicine,Sohag University.
}

\begin{abstract}
Introduction:In recent years, the approach to adjuvant therapy for rectal cancer in the United States and Europe has been shifting toward preoperative therapy to promote sphincter-preserving surgery and decrease acute and late bowel toxicity.

Patients\&Methods:This study will be a phase II trial to study the effectiveness of combining chemotherapy (Gemcitabine) and external - beam radiotherapy followed by surgery in treating patients who have locally advanced rectal cancer, It is carried on Sohag University Hospital \&Sohag NCI since( October 2014- December2017), the dose of radiotherapy 5040 cGy / $28 t t t$, and chemotherapy dose is $\mathbf{4 5} \mathbf{m g} / \mathbf{m} \mathbf{2}$ given twice weekly during radiotherapy sessions only, the study included 30 patients (age 18 and over), having rectal cancer with the following eligibility criteria:histologically proven,previously untreated rectal carcinoma, every patient in the study was subjected to the following:history\& physicalexamination,laboratory investigations, radiological studies.

Results:Thirty patients were included in the study,13 females (43.3\%) and 17 male( $56.6 \%$ ) mean of the age was 49 years. (range24-65year), most of them had adenocarcinoma of the rectum, the main presenting symptom was bleeding per $\operatorname{rectum}(56.7 \%)$, as regard to the response to the neoadjuvanttreatmen $12 / 30$ patients (40\%) achieved Partial response, 8/30patients $(27.6 \%)$ achieved complete response and 10/30 patients (33\%) had no response, abdomino - perineal resection was performed in 19 patients(63.3\%) and 11 patients (36.7\%) underwent sphincter preservation operation, about the toxicity in this study,for chemotherapy diarrhea was the most common manifestation which seen in 25 patients $(83 \%)$,followed by loss of appetite seen in 9 patients(63.3\%), for radiotherapy 19 patients $(63 \%)$ developed wet desquamation. the rate of Overall Survival of the 2-years study is (80\%), the Disease free survival is $(63 \%)$ while the progression free survival is $(72 \%), \mathrm{N}$ stage was the only significant factor affecting the disease free survival and overall survival.

Conclusion: Pre-operative chemo-radiotherapy in dose and fractionation schedule used is safe, effective and well tolerated, allowed sphincter preservation which performed in $\mathbf{3 6 . 7 \%}$ ) of patients
\end{abstract}

\section{Introduction}

There is increasing use of preoperative chemor-adiotherapy as a component of combined-modality treatment (CMT) for clinically resectable rectal cancer.

The aim of this study:Primary Objectives;

1.Determine the Clinical Response Rate in patientswith locally advanced rectal carcinoma treated with preoperative concurrent chemotherapy and radiotherapy.

2.Determine the Pathological response rate in patientswith locally advanced rectal carcinoma treated by surgical resection.

3.Effect of preoperative concurrent chemotherapy andradiotherapy on thepossibility of performingsphincter 
sparing surgery in rectal cancer not suitablefor these procedures.

4.Effect of preoperative chemotherapy and radiotherapyonresectability rates of locally advancedrectal cancer (borderline resectability)

Secondary Objectives;

1.Determine the toxicity of this preoperative regimen in patients with locally advanced rectal carcinoma.

2.Determine the local control, and median overall survival in patients treated with this regimen.

3.Detection of possible prognostic factors and correlating them with response and prognosis in patients treated with this regimen.

\section{Patients and Methods}

This study will be a phase II trial to study the effectiveness of combining chemotherapy (Gemcitabine) and external - beam radiotherapy followed by surgery in treating patients who have locally advanced rectal cancer. It is carried on Sohag University Hospital \&Sohag NCI since( October 2014December2017).

Study Type: Interventional

Study Design: Treatment, NonRandomized, Open Label, Uncontrolled, Single Group Assignment, Safety/Efficacy Study.

OfficialTitle: phase II Study of Preoperative Concurrent Chemotherapy and Radiotherapy in patients with Locally Advanced Rectal Carcinoma.

\section{Eligibility criteria:}

Disease

Characteristics:Pathologically / Histologically proven, previously untreated rectal carcinoma not suitable for primary sphincter sparing surgery that begins within $12 \mathrm{~cm}$ of the anal verge by sigmoidoscopy and / or colonscopy either (1) inoperable or (2) locally advanced,no distant metastatic disease.

\section{Patient Characteristics:}

Age: 18 and over,Performance status:Karnofsky performance status (KPS) $70 \%$ or more,Hematopoietic: adequate B.M.reserve:

Granulocyte count at least $1500 / \mathrm{mm}$,Platelet count at least 100000/mm, Hepatic:Bilirubin not greater than upper limit of normal (ULN),SGOT/SGPT not greater than 2.5times ULN.

Renal :Urea and creatinine not greater than $1.5 \mathrm{mg} / \mathrm{dl}$.

\section{Work up:}

Every patient in the study was subjected to the following:

History \& Physical examination:Including patient's performance status and per rectal digital examination,Laboratory investigation:

Complete blood picture.

Kidney function test (Blood urea, Serum creatinine).

Liver function test (Albumin, Liver enzymes SGOT , SGPT).

Fasting blood sugar level .

Tumor markers : CEA, CA19-9.

Radiological studies:

Chest $\mathrm{x}$-ray posteroanterior and lateral views.

C.T. scans of pelvis and abdomen and/or MRI of pelvis and abdomen.

Barium enema (optional).

Proctoscopy or colonoscopy.

After complete work up, patients were staged before treatment according to TNM Clinical Staging .

2- Treatment Plan:

All patients were treated by preoperative concurrent chemoradiotherapy.

\section{Radiotherapy:}

Target volume: Target volume included the rectum and the draining lymph node chains (pararectal, hypogastric, presacral lymph nodes) up to $\mathrm{S} 1-\mathrm{S} 2$ junction.

Dose and energy: all patients were treated by photon $6 \mathrm{MeV}$ generated from linear accelerator. The dose was 5040 cGy in 28 fractions over5 weeks. 


\section{Chemotherapy:}

Gemcitabine was prescribed at $45 \mathrm{mg} / \mathrm{m} 2$ given twice weekly during radiotherapy sessions only.

Reassessment: $4-6$ weeks after completion of chemo-radiation therapy,all patients will re-evaluated by CBC, CEA, digital rectal examination, CT or MRI of pelvis and abdomen and proctoscopy to evaluate patients for the possibility of surgery.this includes;

a)Re-staging by TNM clinical staging.
b)Assessment of tumor response.

\section{Surrgey:}

After re-staging, all patients were subjected to surgery in the form of abdominally assisted trans-anal coloanal pull through as a sphincter preserving procedure or to abdominoperineal resection.

\section{Toxicity:}

Acute and chronic toxicity was evaluated according to Common Terminology Criteria for Adverse Events(CTCAE).

\section{Results}

The study showed that the mean age of the study patients was $46.8 \pm 10.8$ years, with a range from 24 to 65, a little more than half of the cases were males (17 case, 57\%) with only 13 cases (43\%) were females.

Nearly half of the cases in our study were from urban regions (16 cases, 53\%) and the other half were rural (14 cases, $47 \%$ ).

Regarding the clinical presentation, bleeding per rectum was the most common presenting symptom representing(56.7\%),followed by colic(16.7\%),Diarrhea (13.3\%), Tenesmus(10\%) and vomiting (3.3\%),

According to Karnofsky performance status about (20\%) of patients have PS $70 \%$, while $(80 \%)$ of patients have PS $>70 \%$,

Regarding the staging, the majority of cases were either stage T3 or T4 (40\% each), with only 6 cases were in stage T2 $(20 \%)$.

Most of cases were in stage $\mathrm{N}+$ (positive $\mathrm{LN}$ involvement), with only 7 cases were in stage N0 (23.3\%). In the study of Tey et al., (2017) 90\% .

Regarding tumor localization from anal verge; it was so near $(<5 \mathrm{~cm})$ in $30 \%$ of cases, and so far $(>10 \mathrm{~cm})$ in $20 \%$ of them. The remaining half of cases were in between the two extremes $(5-10 \mathrm{~cm}$ from anal vetage).

Regarding pathology in our study, the most frequent pathology was adenocarcinoma (26 cases), the histologic grading was; MDA, seen in 13 cases WDA ( 7 cases, $23.3 \%$ ), then PDA (6 cases, 20\%), The others were; SRC ( 2 cases, $6.7 \%$ ), and lastly mucoid and mucinous carcinoma (1 cases each) Similarly ; $(43.3 \%)$. This was followed by WDA ( 7 cases, $23.3 \%$ ), then PDA ( 6 cases, 20\%), The others were; SRC ( 2 cases, $6.7 \%$ ), and lastly mucoid and mucinous carcinoma (1 cases each) Similarly.

The most common histologic tumor grade was $\operatorname{MDA}(43.3 \%$,) followed by WDA( $23.3 \%$ ) and lastly PDA(20\%).

As regard to the acute toxicity in our the GIT toxicity grade $1 \& 2$ was in $20 \%$ of patients and grade 3 was in $16 \%$ of the patients .

There were no significant differences between males and females regarding demographic, clinical or therapeutic data. The only two exceptions were clinical presentation (with more bleeding per rectum, vomiting and diarrhea among females and tumor location from the anal verge, where females had nearer tumor localization compared to males.

There was no significant relation between response to chemotherapy and each of age of the patients, sex, disease pathology, tumor stage., while there was significant 
relation between response to chemotherapy and tumor localization, with cases with farer localization tend to be CR more than those with shorter distance from anal verge. We found that there was no significant relation between overall survival of the patients and their demographic and clinical characteristics. The only exception was the $\mathrm{N}$ stage, where patients with negative lymph nodes had longer survival.

\section{Discussion}

Our study showed that the mean age of the study patients was $46.8 \pm 10.8$ years, with a range from 24 to 65 years. Our cases were generally younger than those cases studied by $\boldsymbol{T e y}$ et al., (2017) as $90 \%$ of their cases were older than 50 years old.

In our study, a little more than half of the cases were males (17 case, 57\%) with only 13 cases (43\%) were females. This was similar to the data recorded by Korkolis et al., (2007) where males accounted for $53 \%$ of their cases. Males were over $65 \%$ o Nearly half of the cases in our study were from urban regions (16 cases, $53 \%)$ and the other half were rural (14 cases, 47\%). Regarding the clinical presentation of our study, bleeding per rectum was the most common presenting symptom representing $(56.7 \%)$,followed by colic(16.7\%),Diarrhea

(13.3\%),Tenesmus(10\%) and vomiting $(3.3 \%)$. In our study according to Karnofsky performance status about (20\%) of patients have PS $70 \%$, while $(80 \%)$ of patients have PS > $70 \%$,

In Weber et al;2002 all patients had KPS $>70 \%$, while in Hee Lee $\mathrm{j}$ et al;2002 all patients had KPS $>80 \%$.

Regarding the staging of cancer in our study patients, the majority of cases

were either stage $\mathrm{T} 3$ or T4 (40\% each), with o stage $\mathrm{N}+$ (positive LN involvement), with only 7 cases were in stage N0 (23.3\%). In the study of Tey et al., (2017) 90\% of cases were in T3 stage, with only 1 case had T2 or T4 stage.
Positive lymph nodes were seen in $85 \%$ of their cases. Similarly, the study of Kairevičè et al., (2017).

Regarding tumor localization from anal verge; it was so near $(<5 \mathrm{~cm})$ in $30 \%$ of cases, and so far $(>10 \mathrm{~cm})$ in $20 \%$ of them. The remaining half of cases were in between the two extremes $(5-10 \mathrm{~cm}$ from anal vetage). This was nearly the same as the results of Hess et al., (2017).

As regard to the acute toxicity in our study the GIT toxicity grade $1 \& 2$ was in $20 \%$ of patients and grade 3 was in $16 \%$ of the patients and that was more or less comparable to those reported by Ouf\&Kamel et al; 2002 .

As regard to the surgical complications in our study it seems acceptable,with no post-operative deaths which is similar to Gerard et al;2003 .

Regarding response to CRT, CR was seen in 8 cases $(27 \%)$, with the remaining 22 cases either $\mathrm{PR}$ or stationary (12 and 10 cases, respectively). The study of Korkolis $\boldsymbol{e t}$ al., (2007) showed that the overall response was recorded in 25 patients (84\%). 5 patients $(17 \%)$ had complete response, 8 patients (27\%) showed microscopic residual disease and 12 patients (40\%) had macroscopic residual disease after treatment.

\section{Conclusion}

Pre-operative chemo-radiotherapy in dose and fractionation schedule used in the present study is safe, effective and well tolerated.

Pre-operative chemo-radiotherapy allowed sphincter preservation in the form of Trans-anal abdominally assisted colon-anal pull-through was performed in $(36.7 \%)$ of patients 
And $(63.3 \%)$ of patients underwent Abdomino-Perineal resection.

Pre-operative chemo-radiotherapy allowed curative surgery as survival and regards tumor response to neoadjuvant therapy; which was $26.7 \%$ achieved complete response, $40 \%$ achieved partial response and 33\% are stationary.

the only significant prognostic factor affecting disease free survival and overall survival was $\mathrm{N}$ staging.

The rate of overall survival in the study is $80 \%$, disease free survival is $63 \%$ and progression free survival is $72 \%$.

Regarding response to CRT, CR was seen in 8 cases $(27 \%)$, with the remaining 22 cases either PR or stationary (12 and 10 cases, respectively).

As regard the DFS was achieved for $63 \%$ of patients while the PFS and OS rate was $72 \%$ and $80 \%$ of patients respectively.

There were no significant differences between males and females regarding demographic, clinical or therapeutic data. The only two exceptions were clinical presentation (with more bleeding per rectum,vomiting and diarrhea among females and tumor location from the anal verge, where females had nearer tumor localization compared to males.

There was no significant relation between response to chemotherapy and each of age of the patients, sex, disease pathology, tumor stage., while there was significant relation between response to chemotherapy and tumor localization, with cases with farer localization tend to be $\mathrm{CR}$ more than those with shorter distance from anal verge.

We found that there was no significant relation between overall survival of the patients and their demographic and clinical characteristics. The only exception was the $\mathrm{N}$ stage, where patients with negative lymph nodes had longer survival.

\section{References}

1. Jakobsen A, Ploen J, Vuong T, Appelt A, Lindebjerg J, Rafaelsen SR. Dose-Effect Relationship in Chemoradiotherapy for Locally Advanced Rectal Cancer: A Randomized Trial Comparing Two Radiation Doses. Int J RadiatOncolBiol Phys. 2012 May 15.

2. Kahi CJ, Boland CR, Dominitz JA, Giardiello FM, Johnson DA, Kaltenbach T. Colonoscopy Surveillance After Colorectal Cancer Resection: Recommendations of the US Multi-Society Task Force on Colorectal Cancer. Gastroenterology. 2016 Mar. 150 (3):758-768.e11.

3. Levin B, Lieberman DA, McFarland B . Screening and surveillance for the early detection of colorectal cancer and adenomatous polyps, 2008: a joint guideline from the American Cancer Society, the US Multi-Society Task Force on Colorectal Cancer, and the American College of Radiology. Gastroenterology 2008; 134:1570

4. Meredith KL, Hoffe SE, Shibata D. The multidisciplinary management of rectal cancer. SurgClin North Am. 2009 Feb. 89(1):177-215, ix-x.Mini, E; Nobili, S; Caciagli, B; Landini, I; Mazzei, T (May 2006). "Cellular pharmacology of gemcitabine". Annals of Oncology.

5. Mork ME, You YN, Ying J. High Prevalence of Hereditary Cancer Syndromes in Adolescents and Young Adults With Colorectal Cancer. J ClinOncol 2015; 33:35

6. Klabunde CN, Cronin KA, Breen $\mathbf{N}$. Trends in colorectal cancer test use among vulnerable populations in the United States. Cancer Epidemiol Biomarkers Prev 2011; 20:161

7. Wu S, Feng B, Li K. Fish consumption and colorectal cancer risk in humans: a systematic review and meta-analysis. Am J Med 2012; 125:551

8. Y Kanemitsu, T. Kato, T. Hirai et al., "Survival after curative resection for mucinous adenocarcinoma of the 
colorectum," Diseases of the Colon and Rectum, vol. 46, no. 2, pp. 160-167 2003

9. van Helmond $\mathbf{J}$, Beart RW. Cancer of the rectum: Operative management and adjuvant therapy. Current Therapy in Colon and Rectal Surgery. 2nd ed. Philadelphia, Pa: Mosby; 2005. 